\title{
Extracellular ubiquitin promotes hepatoma metastasis by mediating M2 macrophage polarization via the activation of the CXCR4/ERK signaling pathway
}

\author{
Jiajing Cai ${ }^{1 \#}$, Qi Zhang ${ }^{1 \#}$, Xuemeng Qian ${ }^{1}$, Jingdong Li $^{2}$, Qi Qi ${ }^{1}$, Ru Sun ${ }^{3}$, Jia Han ${ }^{1}$, Xinfang Zhu ${ }^{1}$, \\ Mengyi Xie ${ }^{2}$, Xiaolan Guo ${ }^{3,4}$, Rong Xia ${ }^{1}$ \\ ${ }^{1}$ Department of Transfusion Medicine, Huashan Hospital, Fudan University, Shanghai, China; ${ }^{2}$ Department of General Surgery, ${ }^{3}$ Department of \\ Clinical Laboratory, Affiliated Hospital of North Sichuan Medical College, Nanchong, China; ${ }^{4}$ Translational Medicine Research Center, North \\ Sichuan Medical College, Nanchong, China \\ Contributions: (I) Conception and design: X Guo, R Xia; (II) Administrative support: J Han, X Zhu, Q Qi; (III) Provision of study materials or \\ patients: J Li, M Xie, R Sun; (IV) Collection and assembly of data: J Cai, Q Zhang; (V) Data analysis and interpretation: J Cai, Q Zhang, X Qian; (VI) \\ Manuscript writing: All authors; (VII) Final approval of manuscript: All authors. \\ "These authors contributed equally to this work. \\ Correspondence to: Xiaolan Guo. Department of Clinical Laboratory, Affiliated Hospital of North Sichuan Medical College, 63 Wenhua Road, \\ Nanchong 637000, China. Email: alan5200gxl@sina.com; Rong Xia. Department of Transfusion Medicine, Huashan Hospital, Fudan University, 12 \\ Urumqi Middle Road, Shanghai 200040, China. Email: xiaronghs@126.com.
}

Background: Stored red blood cell (RBC) transfusion has been shown to enhance the risk of cancer recurrence. However, the underlying mechanism remains unknown. At our lab, we have demonstrated that the extracellular ubiquitin (eUb) released by aged RBCs could promote tumor metastasis in a melanoma mouse model. This study aimed to confirm the pro-tumor effect of eUb on hepatocellular carcinoma (HCC) and explore the related immunoregulatory mechanisms.

Methods: Forty HCC tissue specimens and the corresponding adjacent nontumor and normal liver tissues were collected. Two human hepatoma cell lines (MHCC-97H and HepG2.2.15), one murine hepatoma cell line (Hepa1-6), and one human monocyte cell line (THP-1) were adopted in this study. The coculture of hepatoma cells with macrophages was initiated with Transwell inserts. Cell migration in vitro was detected by Transwell and wound-healing assays, while in vivo tumor metastasis was measured by luciferase assay and H\&E staining. Macrophage polarization was measured by flow cytometry, immunofluorescence, ELISA, qPCR, and Western blot. Protein expression was detected by Western blot, and immunoprecipitation was used to confirm the interaction between $\mathrm{Ub}$ and CXCR4 (CXC chemokine receptor type 4).

Results: Ub and CXCR4 were significantly upregulated in HCC tissues, and a positive correlation existed between them. In vitro, the migration of hepatoma cells was not affected by eUb directly, but their metastatic abilities were enhanced after coculture with the macrophages pretreated with eUb. Meanwhile, eUb promoted hepatoma cell metastasis in the lung in vivo and increased the ratio of M2 macrophages in the lung tissues and peripheral blood of tumor-bearing mice. Furthermore, the eUb-induced M2 macrophage polarization was related to the activation of the CXCR4/ERK (extracellular regulated protein kinase) signaling pathway.

Conclusions: Extracellular ubiquitin promoted hepatoma metastasis through M2 macrophage polarization via the activation of the CXCR4/ERK signaling pathway, indicating that a personalized transfusion strategy is needed for the treatment of HCC patients. Neutralizing Ub in stored RBC units could lessen the detrimental clinical outcomes induced by the transfusion of stored RBCs.

Keywords: Ubiquitin; metastasis; macrophage polarization; hepatoma; CXCR4/ERK pathway

Submitted Jan 28, 2020. Accepted for publication Jul 01, 2020.

doi: $10.21037 /$ atm-20-1054

View this article at: http://dx.doi.org/10.21037/atm-20-1054 


\section{Introduction}

Transfusion is a vital treatment for cancer patients, especially for those with hepatocellular carcinoma (HCC), due to chronic blood loss. However, the safety of transfusion is controversial. Allogeneic blood transfusion can induce transfusion-related immunomodulation (TRIM) responses, including incompatibility issues, allergic reaction, coagulopathy, postoperative infection, multiple organ failure, and cancer recurrence (1-3). These complicated physiologic responses may be induced by apoptotic cells, residual leukocytes, and biological molecules such as soluble mediators, HLA peptides, and cytokines contained in the RBC units (4).

Stored red blood cell (RBC) transfusion has been demonstrated to enhance the risk of recurrence and mortality in multiple cancers (5-9), although its underlying mechanism is still unknown. Ubiquitin ( $\mathrm{Ub}$ ) is a highly conserved $8.5 \mathrm{kDa}$ protein that can be found in all eukaryotes, and its concentration in RBCs is much higher than in other cells (10-12). After 35 days of storage, the hemolysis that occurs in the storage of RBC units has been found to significantly increase the concentration of extracellular ubiquitin $(\mathrm{eUb})$ in whole blood $(13,14)$. The most important function of intracellular $\mathrm{Ub}$ is degrading proteins via the ubiquitin-proteasome system (15). The Ub-mediated posttranslational modifications of misfolded and damaged proteins play crucial roles in multiple cellular processes. To date, the effects of intracellular Ub on the regulation of intracellular transformations have been investigated to a relatively considerable degree, but less is known about the functions of extracellular Ub. At our lab, we have demonstrated that $\mathrm{eUb}$ can exert immunoregulation effects, such as reinforcing the suppressive effect of Tregs (regulatory $\mathrm{T}$ cells), promoting Th cell differentiation into the immunosuppressive Th2 phenotype, and affecting cytokine secretion $(14,16)$.

$\mathrm{eUb}$ has been reported to exert pleiotropic functions, including immunoregulatory, anti-inflammatory, and neuroprotective effects, and to regulate the growth and apoptosis of hematopoietic cells and modulate lymphocyte differentiation (17-21). However, studies aimed at investigating the relationship between eUb and the development of cancer are limited. Recently, we have demonstrated that eUb might be the link between allogeneic blood transfusion and poor cancer prognosis; in the melanoma mouse model, eUb promoted tumor metastasis, which might be related to eUbmediated immunomodulation (22). However, the specific immunoregulatory mechanisms of eUb need to be elucidated in depth. Therefore, this study aimed to confirm the pro-tumor effect of eUb on HCC in vitro and in vivo and to explore the effects of eUb on the regulation of immunocytes.

We present the following article in accordance with the ARRIVE reporting checklist (available at http://dx.doi. org/10.21037/atm-20-1054).

\section{Methods}

\section{Patient specimens}

HCC tissues and the corresponding adjacent nontumor and normal liver tissues were obtained from 40 HCC patients who had undergone surgical resection at the Affiliated Hospital of North Sichuan Medical College. This study was conducted in accordance with the Declaration of Helsinki (as revised in 2013), and the protocol was approved by the Ethics Committee of the Affiliated Hospital of North Sichuan Medical College (No. 2018-EA-028). Informed consent was obtained from each patient. The clinicopathological features of the HCC patients are shown in Table 1.

\section{Cells and reagents}

Human hepatoma cell lines HepG2.2.15 and MHCC-97H, mouse hepatoma cell line Hepa1-6, and human monocyte cell line THP-1 were purchased from Shanghai Institute of Cell Biology, Chinese Academy of Sciences (Shanghai, China). HepG2.2.15, MHCC-97H, and Hepa1-6 cells were cultured in Dulbecco's Modified Eagle Medium (DMEM) containing $10 \%$ fetal bovine serum (both Hyclone; GE Healthcare Life Sciences, Logan, UT, USA), penicillin $(100 \mathrm{U} / \mathrm{mL})$, and streptomycin $(100 \mu \mathrm{g} / \mathrm{mL}$; Sigma-Aldrich; Merck KGaA, Darmstadt, Germany). The THP-1 cells were maintained in RPMI 1640 medium containing 10\% FBS (fetal bovine serum) and penicillin/streptomycin. Cells were incubated in a humidified incubator at $37^{\circ} \mathrm{C}$ under 5\% $\mathrm{CO}_{2}$. Ub (\#U6253), PMA (\#P1585), AMD3100 (\#A5602), and U0126 (\#U120) were purchased from Sigma-Aldrich (St. Louis, MO, USA). All antibodies including mTOR(\#2983), p-mTOR (\#5536), Akt (\#4685), p-Akt (\#4060), Vimentin (\#5741), E-cadherin (\#3195), N-cadherin (\#13116), Erk1/2 (\#4695), p-Erk1/2 (\#4370), Arg-1 (\#93668), and GAPDH (\#2118) were obtained from Cell Signaling Technology (Beverly, MA, USA). MMP-9 (\#76003), iNOS (\#178945), TGF- $\beta$ (\#179695), CXCR4 
Table 1 The clinicopathological features of HCC patients

\begin{tabular}{lc}
\hline Parameters & Values \\
\hline Age, mean \pm SD & $54.78 \pm 12.07$ \\
Gender, n (\%) & $33(82.5)$ \\
Male & $7(17.5)$ \\
Female & \\
HBsAg, n (\%) & $27(67.5)$ \\
+ & $13(32.5)$ \\
- & \\
Cirrhosis, n (\%) & $24(60.0)$ \\
+ & $16(40.0)$ \\
- & \\
Tumor size, n (\%) & $12(30.0)$ \\
$<5$ cm & $28(70.0)$ \\
$\geq 5$ cm & \\
PVTT, n (\%) & \\
IIIIV & $6(15.0)$ \\
\hline
\end{tabular}

HCC, hepatocellular carcinoma ;PVTT, portal vein tumor thrombus.

(\#181020/124824) and Ub (\#7780) were obtained from Abcam (Cambridge, MA, USA).

\section{Animals}

Male C57BL/6 mice aged 6 to 8 weeks were purchased from Shanghai Research Center for Southern Model Organisms (Shanghai, China). All animal experiments in this study were approved by the Laboratory Animal Care and Use Committee of Fudan University (No. 2019 Huashan Hospital JS-259) and conducted under the "Guide for the Care and Use of Laboratory Animals" recommended by the US National Institutes of Health.

\section{Tumor metastasis model}

Lung metastasis models were established via injection of Hepa 1-6-luciferase cells $\left(2 \times 10^{6}\right)$ suspended in $300 \mu \mathrm{L}$ PBS into the lateral tail vein of the mice. The mice were randomly divided into two groups: the eUb group and the control group. Intraperitoneal injections of eUb (U5507; Sigma-Aldrich, St. Louis, MO, USA) and physiological saline were performed 3 times at $24 \mathrm{~h}$ intervals. After three weeks of feeding, the tumor metastasis was monitored using the IVIS Spectrum CT (PerkinElmer, Waltham, Massachusetts, USA).

\section{Coculture of hepatoma cells with macrophages}

THP-1 cells $\left(1 \times 10^{6}\right)$ were seeded in Transwell inserts (\#3412; Corning, NY, USA) and pretreated with PMA (100 nM) for $24 \mathrm{~h}$ to induce $\mathrm{M} 0$ macrophages. Next, Ub $(10 \mu \mathrm{g} / \mathrm{mL})$ was used to treat M0 macrophages for $72 \mathrm{~h}$. Meanwhile, HepG2.2.15 and MHCC-97H cells $\left(1 \times 10^{6}\right)$ were seeded in 6-well plates for $24 \mathrm{~h}$. Then, the Transwell inserts were put into the 6-well plates to enable coculture. After $72 \mathrm{~h}$ of coculture, the HepG2.2.15 and MHCC-97H cells were collected for analysis.

\section{Transwell assay}

Cells $\left(5 \times 10^{4}\right)$ suspended in DMEM medium containing $5 \%$ FBS were seeded in the upper chamber of Transwell inserts (\#3422; Corning, NY, USA). DMEM supplemented with $20 \% \mathrm{FBS}$ was added to the lower chamber as an attractant solution. After incubation, the remaining cells on the upper chamber were cleared, and the cells that had migrated to the underside of the chamber were fixed with methanol and stained with Giemsa. The migration ability of the cells was measured based on the number of cells that had migrated to the underside of the chamber. Images were captured with an inverted microscope (Olympus, Tokyo, Japan).

\section{Wound-bealing assay}

Cells $\left(5 \times 10^{5}\right)$ were seeded in 6 -well plates and cultured until a confluent monolayer was formed. A scratch was made with a $10 \mu \mathrm{L}$ pipette tip. The medium was removed, and the cultures were washed twice with PBS to remove the floating cells. The cells were then cultured in medium containing $5 \%$ FBS. The migration ability of the cells was detected by the closure of the scratch. Images were captured with an inverted microscope.

\section{Flow cytometry}

Cells $\left(1 \times 10^{6}\right)$ were suspended in PBS and preincubated with 
CD86 antibody for $30 \mathrm{~min}$ at $4{ }^{\circ} \mathrm{C}$. Next, for intracellular CD206 and CD68 staining, cells were fixed for $15 \mathrm{~min}$ with a fixation buffer before pre-cooled $50 \%$ methanol was added for $10 \mathrm{~min}$ at $4{ }^{\circ} \mathrm{C}$. Then, the cells were washed in PBS with $5 \% \mathrm{FBS}$ and resuspended with permeabilization buffer before co-staining with the CD206 and CD68 antibodies at RT for $30 \mathrm{~min}$. Finally, the cells were washed and resuspended in PBS, and flow cytometry was performed. The peripheral blood of the mice was collected, and after the supernatants were removed by centrifugation, the ratios of macrophages and Tregs were measured by staining with CD4, CD25, Foxp3 ${ }^{+}, \mathrm{F} 4 / 80$, CD11b, CD206, and CD80 antibodies (BioLegend, San Diego, CA, USA), according to the above protocols.

\section{Enzyme-linked immunosorbent assay (ELISA)}

The cytokine content was measured using ELISA kits (R\&D Systems, Minneapolis, MN, USA). Blood was harvested from the anesthetized mice and centrifuged for $5 \mathrm{~min}$ at $3,500 \mathrm{r} / \mathrm{min}$ to collect the serum. Meanwhile, the supernatants from the cell culture medium were stored at $-80{ }^{\circ} \mathrm{C}$ pending assay. ELISA assay was performed according to the manufacturer's instructions, with each sample detected in triplicate. The concentration of cytokines was calculated using a standard curve.

\section{Immunoprecipitation}

Cell lysates were incubated with $\mathrm{Ub}$ antibody and normal rabbit IgG overnight at $4{ }^{\circ} \mathrm{C}$ and further incubated with agarose (Sigma-Aldrich, St. Louis, MO, USA) for $3 \mathrm{~h}$ on a rocking platform. Beads were collected through centrifugation at $12,000 \times \mathrm{g}$ for $1 \mathrm{~min}$ at $4{ }^{\circ} \mathrm{C}$, rinsed in wash buffer, and then resuspended in SDS loading buffer. Finally, the lysates were subjected to SDS-PAGE, transferred to PVDF membrane, and detected by immunoblotting with the corresponding antibody.

\section{Hematoxylin and eosin staining}

The lung tissues were fixed in $4 \%$ formaldehyde and embedded in paraffin. The tissue sections of $5 \mathrm{~mm}$ thickness were deparaffinized in xylene, rehydrated in graded alcohol solution, and then stained with hematoxylin and eosin according to the protocol. Images were captured using a light microscope (Olympus, Tokyo, Japan).

\section{Immunofluorescence}

The lung tissues were harvested and incubated with CD206 antibody overnight at $4{ }^{\circ} \mathrm{C}$. After, the tissues were washed and incubated $1 \mathrm{~h}$ with FITC goat anti-mouse IgG secondary antibody at $37^{\circ} \mathrm{C}$ in the dark. Nuclei were stained with DAPI for $10 \mathrm{~min}$. Images were captured using an inverted fluorescence microscope (Olympus, Tokyo, Japan).

\section{$q P C R$}

Total RNA was extracted with TRIzol (Invitrogen, California, USA) and reverse transcribed to cDNA by PrimeScript ${ }^{\mathrm{TM}}$ Reverse Transcriptase (Takara Bio, Shiga, Japan). qPCR was performed with SYBR ${ }^{\circledR}$ Premix Ex $\mathrm{Taq}^{\mathrm{TM}}$ II (Takara Bio, Shiga, Japan) and the LightCycler ${ }^{\circledR}$ 480 System (Roche, Basel, Switzerland). The method of $2^{-\Delta \Delta C t}$ was used to analyze the mRNA level. The relative expressions of all genes were quantified as fold changes versus control. GAPDH (Glyceraldehyde-3-phosphate dehydrogenase) was used as internal control. Table 2 shows the primers used for qPCR.

\section{Western blot}

Cells were washed with cold PBS, and the extracts were prepared using lysis buffer. The lung tissues from the mice were also prepared. The protein extracts were subjected to SDS-PAGE and transferred to PVDF membranes. The membranes were blocked with 5\% BSA for $1 \mathrm{~h}$ at RT and then incubated overnight using primary antibody at $4{ }^{\circ} \mathrm{C}$. After that, the membranes were washed and incubated with the second antibody conjugated to horseradish peroxidase at RT for $1 \mathrm{~h}$. The protein signal was checked with Fusion FX7 (VILBER, Paris, France).

\section{Statistical analysis}

GraphPad Prism 7 (GraphPad Software, La Jolla, CA) was used to perform statistical analysis. Non-parametric data (the expressions of CXCR4 and Ub in clinical tissues) were shown as median $\left(25^{\text {th }}\right.$ to $75^{\text {th }}$ interquartile range), and comparisons between groups were performed with Wilcoxon matched-pairs signed rank test and KruskalWallis test. Correlation of non-parametric data were analyzed using Spearman Correlation test. Parametric data were expressed as mean \pm standard deviation (SD) 
Table 2 List of primers used for qPCR

\begin{tabular}{lll}
\hline Gene & Forward & Reverse \\
\hline CD80 & AAACTCGCATCTACTGGCAAA & GGTTCTTGTACTCGGGCCATA \\
CD163 & GCGGGAGAGTGGAAGTAAAG & GTTACAAATCACAGAGACCGCT \\
CD206 & CTACAAGGGATCGGGTTATGGA & TTGGCATTGCCTAGTAGCGTA \\
CCL3 & GCTGACTACTTTGAGACGAGCA & ATATTTCTGGACCCACTCCTCA \\
CCL18 & GCTGCCTCGTCTATACCTCCT & GGTCGCTGATGTATTCTGGA \\
CCL22 & TGATTACGATCCGTTACCGTCT & CCTGAAGGTTAGCAACACCAC \\
$i N O S$ & ACGTGCGTTACTCCACCAACAA & CATAGCGGATGAGCTGAGCATT \\
IL-10 & AGAACCTGAAGACCCTCAGGC & CCACGGCCTTGCTCTTGTT \\
$T G F-\beta$ & GAGGGGAATTGAGGGCTTT & CGGTAGTGAACCCGTTGATG \\
$T N F-\alpha$ & TAGCCCATGTTGTAGCAAACC & ATGAGGTACAGGCCCTCTGAT
\end{tabular}

and comparisons between groups were performed with Student's $t$-test and one-way ANOVA. A P value of $<0.05$ was considered statistically significant.

\section{Results}

Ub was upregulated in HCC tissues and positively related with CXCR4

Since eUb was confirmed to promote tumor metastasis in a melanoma mouse model in our previous study, we wanted to explore further the role of $\mathrm{Ub}$ in the development and progression of HCC. First, we examined Ub expression in human HCC tissues and the corresponding adjacent nontumor and normal liver tissues by Western blot. The results showed that the expressions of $\mathrm{Ub}$ and CXCR4 were strikingly stronger in HCC tissues than in adjacent nontumor and normal liver tissues (Figure 1A), and there was no significant difference between their expressions in adjacent nontumor tissues and normal liver tissues (Figure $1 B, C$ ). Moreover, there was a positive correlation between $\mathrm{Ub}$ and CXCR4, and their correlation in HCC tissues was stronger than in adjacent nontumor and normal liver tissues (Figure 1D). Together, these results indicated that $\mathrm{Ub}$ might participate in the development and progression of HCC.

\section{eUb had no significant effect on the migration of hepatoma cells}

To determine whether eUb participates in the development and progression of $\mathrm{HCC}$, the effect of eUb on the migration ability of hepatoma cells (MHCC-97H and HepG2.2.15) was observed in vitro. After treatment with eUb $(10 \mu \mathrm{g} / \mathrm{mL})$ for $48 \mathrm{~h}$, the Transwell assay showed that eUb did not significantly affect the mobility of $97 \mathrm{H}$ and HepG2.2.15 (Figure 2A). The same result was obtained from the woundhealing assay (Figure 2B). We also detected the expressions of Vimentin and E-cadherin, which are the key proteins in epithelial-mesenchymal transition, as well as those of MMP9 and TGF- $\beta$, which are related to tumor metastasis. The data showed no obvious changes in the expressions of the proteins. Meanwhile, CXCR4, which is involved in cancer metastasis, was not significantly changed by eUb exposure (Figure 2C). Collectively, the above results demonstrated that eUb had no direct effect on the migration of hepatoma cells in vitro.

\section{eUb enhanced the migration of hepatoma cells via the involvement of macrophages}

Tumor-related macrophages have been shown to participate in tumor development and progression, and eUb possibly regulates the differentiation and function of immunocytes. Therefore, we investigated whether eUb could affect the migration of hepatoma cells via the involvement of macrophages. Since the differentiation of THP-1 monocytes has been used as a macrophage model in vitro, THP-1 cells were differentiated into macrophages through incubation with PMA. After $24 \mathrm{~h}$, the THP-1 cells were adherent and were designated as $\mathrm{M} 0$ phenotype macrophages. The M0 macrophages were treated with 
A

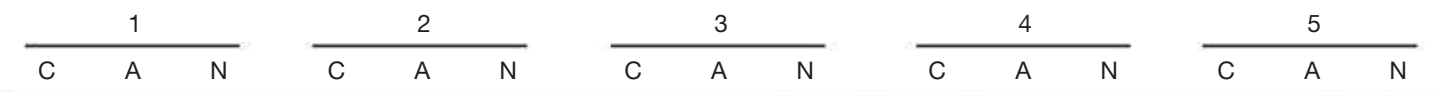
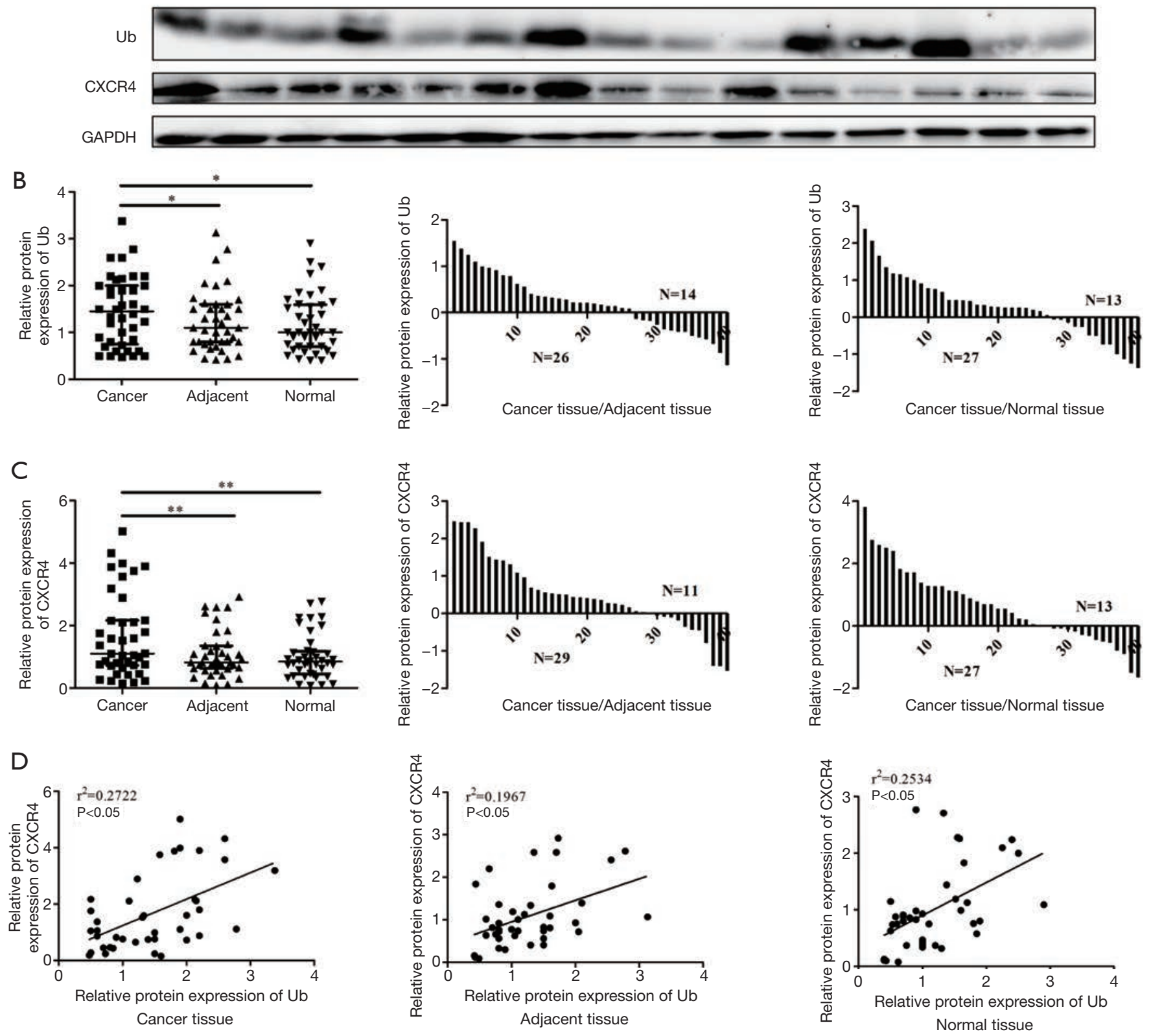

Figure $1 \mathrm{Ub}$ was upregulated in HCC tissues and positively related to CXCR4. (A,B,C) The expressions of Ub and CXCR4 in HCC tissues and their corresponding adjacent nontumor and normal liver tissues were detected by Western blot. (D) The correlation between Ub and CXCR4 was analyzed by linear regression. GAPDH was used as a loading control. 1-5: patient number. C, cancer tissue; A, adjacent nontumor tissue; $\mathrm{N}$, normal liver tissue. ${ }^{*}, \mathrm{P}<0.05$; ${ }^{* *}, \mathrm{P}<0.01$. 


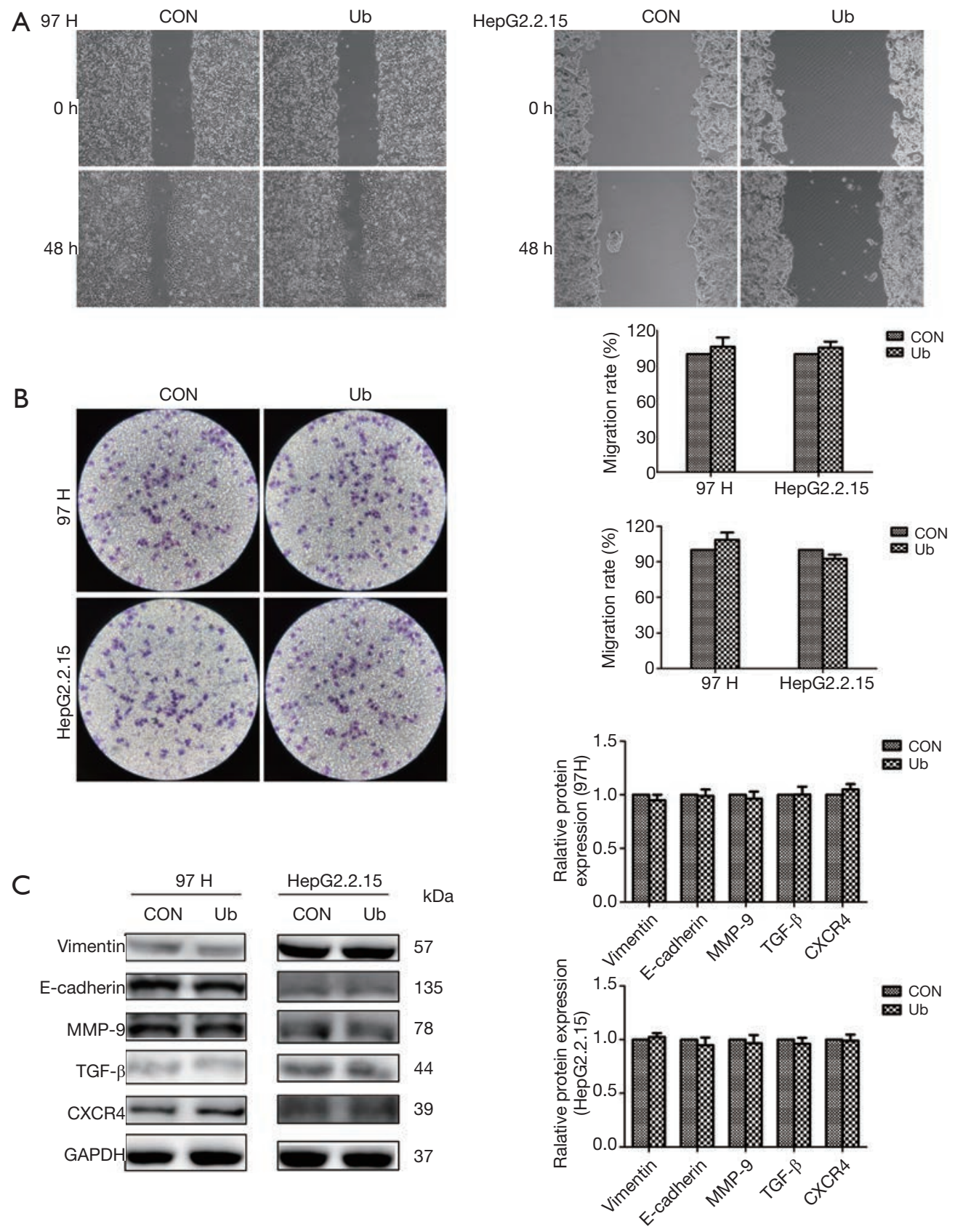

Figure $2 \mathrm{eUb}$ had no significant effect on the migration of hepatoma cells. (A,B) 97H and HepG2.2.15 cells were treated with eUb $(10 \mu \mathrm{g} / \mathrm{mL})$ for $48 \mathrm{~h}$, Transwell and wound-healing assays were performed to measure cell migration ability $(\times 200)$. (C) The expressions of proteins related to tumor metastasis were detected by Western blot. GAPDH was used as a loading control. CON, control. 
eUb $(10 \mu \mathrm{g} / \mathrm{mL})$ for $72 \mathrm{~h}$, and then cocultured with $97 \mathrm{H}$ and HepG2.2.15 cells via Transwell inserts. The migration abilities of the $97 \mathrm{H}$ and HepG2.2.15 cells were measured after $72 \mathrm{~h}$ coculture. The results showed that the mobility of hepatoma cells was increased after coculture with macrophages pretreated with eUb compared to that of the hepatoma cells cocultured with the control macrophages treated with physiological saline (Figure $3 A, B$ ). Besides, the expressions of Vimentin, E-cadherin, MMP-9, TGF- $\beta$, and CXCR4 were elevated simultaneously (Figure 3C). These findings indicated that eUb could enhance the migration of hepatoma cells via the involvement of macrophages.

\section{eUb promoted the metastasis of hepatoma cells in the tumor-bearing model}

A murine lung metastasis model was established to investigate whether eUb promoted the metastasis of hepatoma cells in vivo. After three weeks of feeding, the bioluminescent signal was much stronger in the lungs of the eUb-treated mice than in those of the control mice (Figure $4 A$ ). Furthermore, a greater number of metastatic nodules were found in the lungs of the Ub-treated mice than in those of the control mice through $\mathrm{H} \& \mathrm{E}$ staining (Figure 4B). Meanwhile, we harvested the lung tissues to detect the expressions of metastasis-related proteins and found that E-cadherin was downregulated; however, Vimentin, N-cadherin, and CXCR4 were upregulated in the eUb group compared to the control group. Moreover, the expressions of p-mTOR and p-Akt, which are related to tumor proliferation, were significantly upregulated in the eUb-treated mice (Figure 4C). These results, taken together, suggested that $\mathrm{eUb}$ promoted the metastasis of hepatoma cells in the tumor-bearing model.

\section{eUb upregulated the ratio of M2 macrophages in tumor- bearing mice}

To determine whether the eUb-induced metastasis of hepatoma cells in vivo was correlated with the M2 macrophage polarization, we collected the peripheral blood of the tumor-bearing mice and analyzed the ratio and polarization of macrophages by flow cytometry. The data showed that the percentages of M0 and M1 macrophages were not obviously changed, while the ratio of M2 macrophages was significantly elevated in the eUb-treated mice (Figure $5 A$ ). We also measured the percentages of $\mathrm{CD}^{+}$lymphocytes and Tregs, which were not significantly changed after eUb intervention (Figure 5B). Meanwhile, the immunofluorescence assay demonstrated that the proportion of M2 macrophages in lung tissues was increased in the eUb-treated group (Figure 5C). The concentration of TGF- $\beta$ in the serum of the eUb-treated mice was upregulated; however, the concentrations of TNF- $\alpha$ and IL10 were not obviously changed compared with the control mice (Figure 5D). Furthermore, the results of Western blot showed that iNOS (M1 macrophage marker) and Arg-1 (M2 macrophage marker) were both enhanced upon eUb exposure; yet, Arg-1 was more significantly upregulated than iNOS (Figure 5E). These data taken together indicated that eUb upregulated the ratio of M2 macrophages in tumor-bearing mice.

\section{eUb promoted M2 macrophage polarization}

Since coculture with eUb-treated macrophages promoted the migration of hepatoma cells, and macrophage polarization could affect the development and progression of cancer, we specifically explored the effect of eUb on macrophage polarization. The M0 macrophages differentiated from THP-1 cells were treated with different concentrations of eUb $(1,2,5$, and $10 \mu \mathrm{g} / \mathrm{mL})$ for $72 \mathrm{~h}$, and we assessed the polarization of the macrophages by measuring the mRNA levels of several classical macrophage markers using qPCR. The expressions of M1 markers (TNF- $\alpha$, iNOS, CD80, and CCL3) were significantly downregulated by eUb; however, the expressions of M2 markers (CD163, CD206, CCL22, IL-10, TGF- $\beta$, and CCL18) were increased (Figure 6A). The expressions of CD86 (M1 macrophage marker) and CD206 (M2 macrophage marker) were also measured by flow cytometry. CD206 expression was obviously increased while there was no change in CD86 expression after eUb exposure (Figure 6B). From these results, we concluded that eUb promoted macrophages polarization towards the M2 phenotype.

\section{eUb might promote M2 macrophage polarization via the CXCR4/ERK pathway}

In the subsequent experiments, we explored the specific mechanisms by which eUb promoted M2 macrophage polarization. We confirmed the binding of $\mathrm{Ub}$ and CXCR4 (Figure $7 A$ ) and observed the upregulation of both CXCR4 and p-Erk1/2 in macrophages after treatment with eUb (Figure 7B). Meanwhile, the expressions of CXCR4 and 
A 97
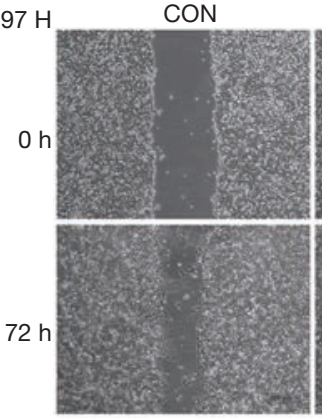

B

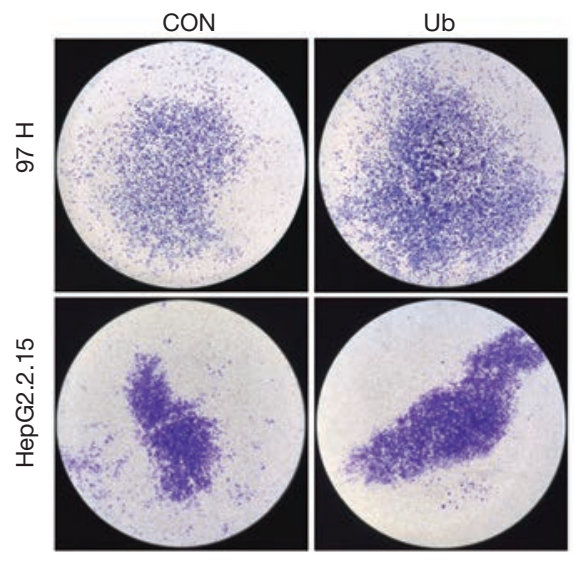

C

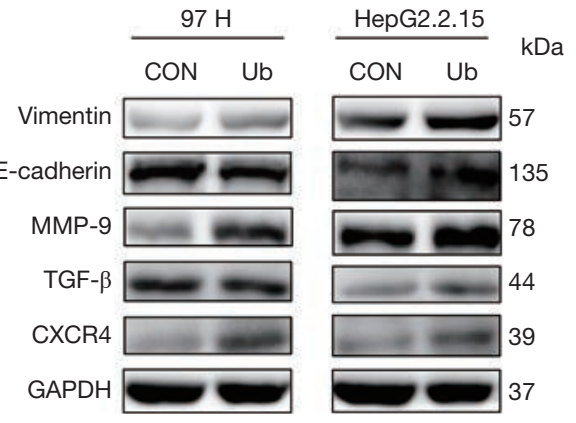

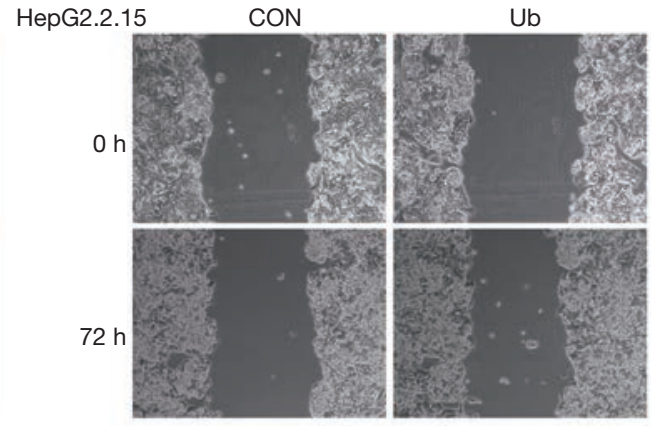
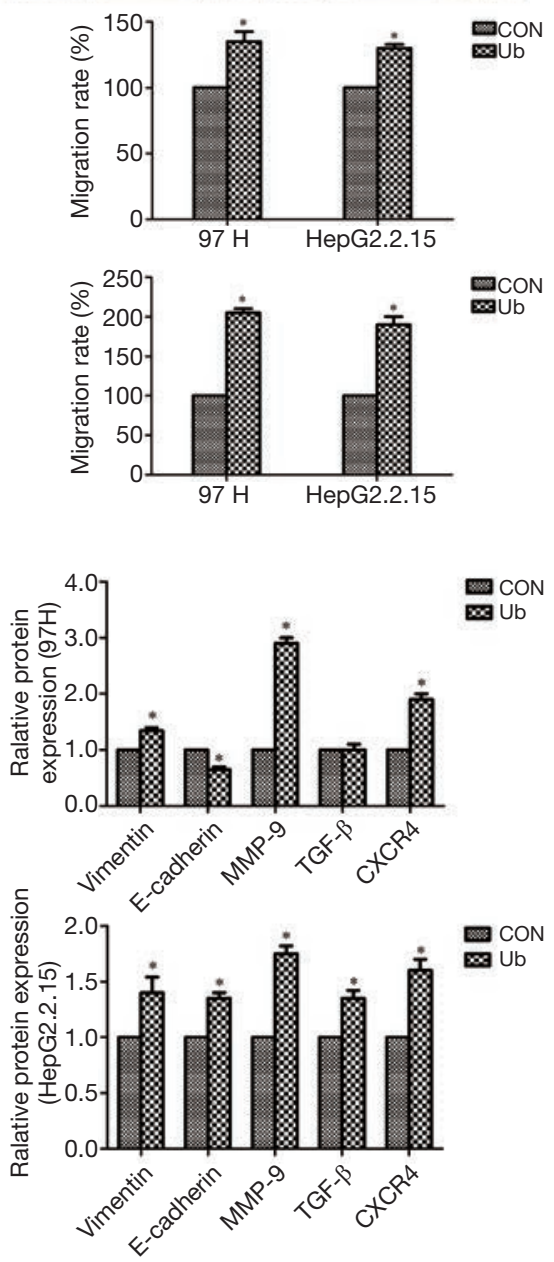

Figure $3 \mathrm{eUb}$ enhanced the migration of hepatoma cells via the involvement of macrophages. THP-1 monocytes were preincubated by PMA $(100 \mathrm{nM})$ for $24 \mathrm{~h}$ to induce M0 macrophages, and then the M0 macrophages were treated with eUb $(10 \mu \mathrm{g} / \mathrm{mL})$ for $72 \mathrm{~h}$. After that, $97 \mathrm{H}$ and HepG2.2.15 cells were cocultured with the macrophages pretreated by eUb via Transwell inserts (pore size, $0.4 \mu \mathrm{m}$ ). After incubation for $72 \mathrm{~h}$, the migration abilities of $97 \mathrm{H}$ and HepG2.2.15 cells were measured by Transwell (A) and wound-healing assay (B, $\times 200$ ). (C) Western blot was used to detect the expressions of key proteins related to tumor metastasis. GAPDH was used as a loading control. *, $\mathrm{P}<0.05$. CON, control. 

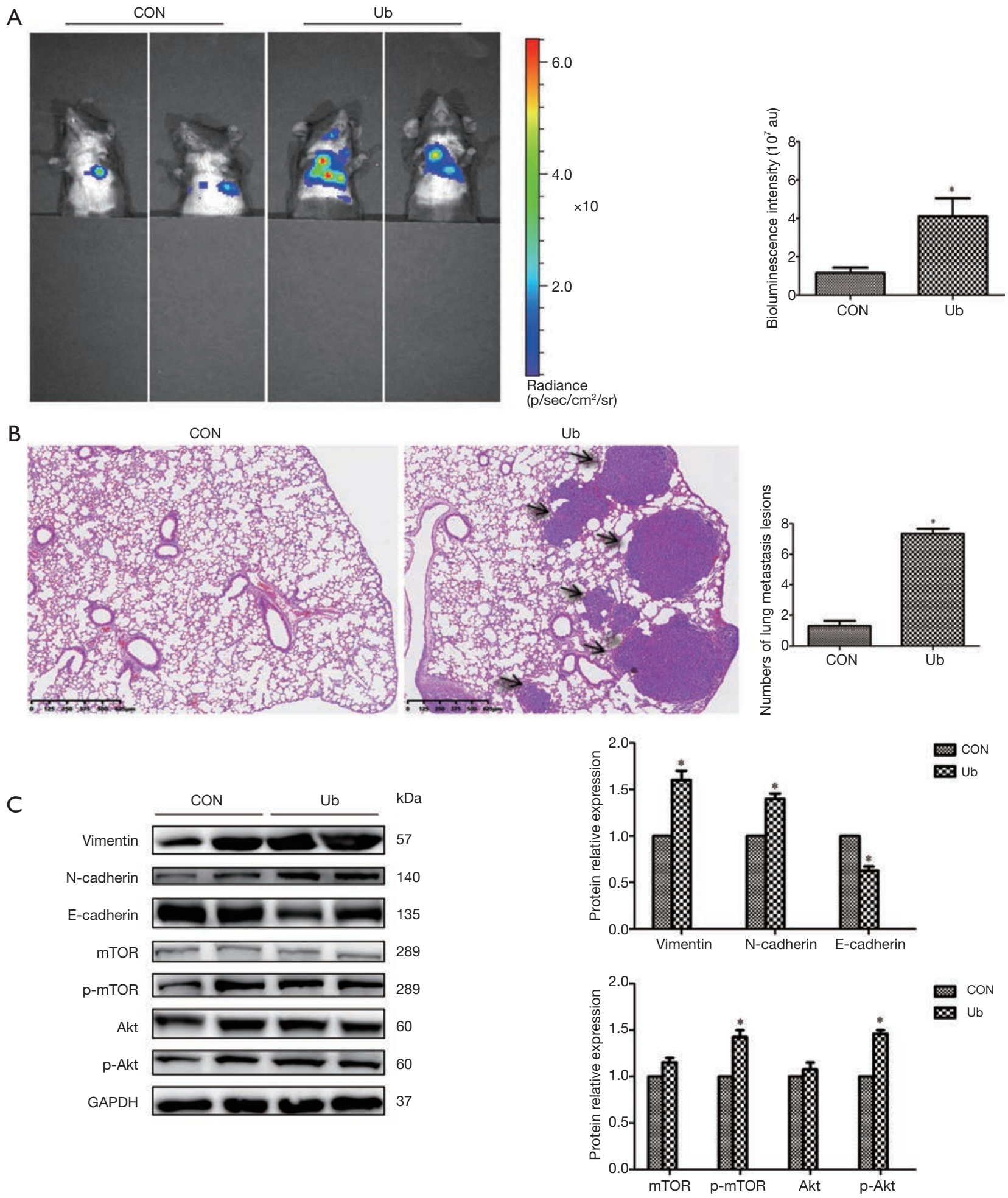

Figure $4 \mathrm{eUb}$ promoted the metastasis of hepatoma cells in the tumor-bearing model. The mice were intraperitoneally injected with eUb (Ub group: $1.6 \mu \mathrm{g} / \mathrm{mL}$ ) or normal saline (CON, control group) after Hepa1-6-luciferase cells were injected into the tail vein of mice. $(\mathrm{A}, \mathrm{B})$ Tumor metastasis was detected by bioluminescent signal and H\&E staining, and the arrows indicated the tumor metastases. (C) The lung tissues of the mice were harvested to detect the proteins related to tumor metastasis through Western blot. GAPDH was used as a loading control. *, $\mathrm{P}<0.05$. CON, control. 
A
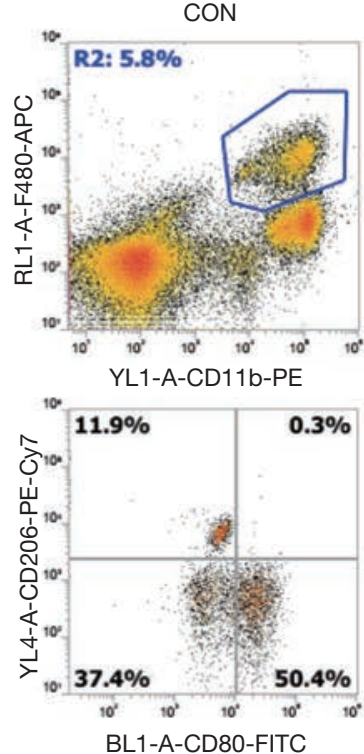

C
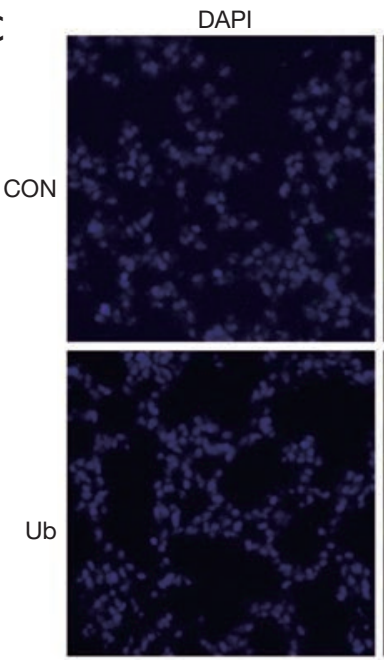

D

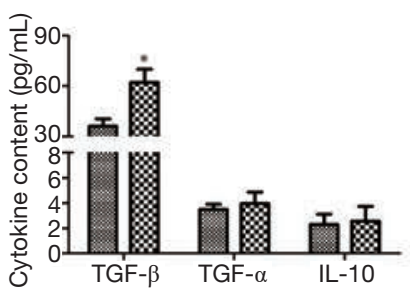

Ub
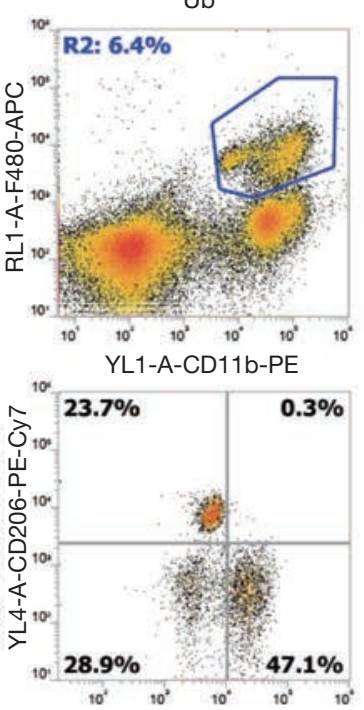

BL1-A-CD80-FITC
B

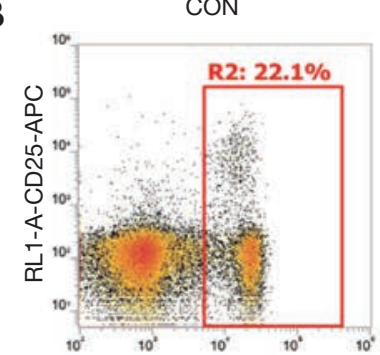

BL1-A-CD4-FITC

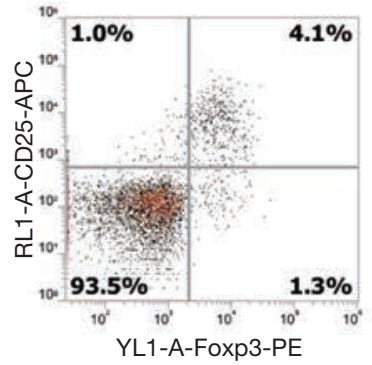

$\mathrm{Ub}$

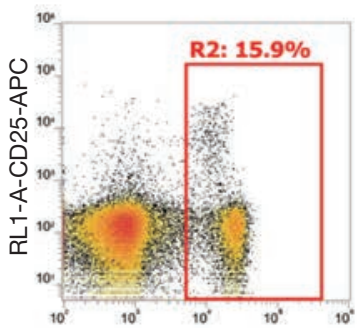

BL1-A-CD4-FITC

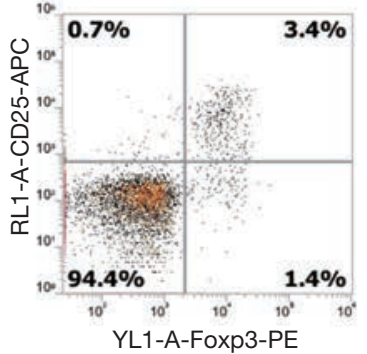

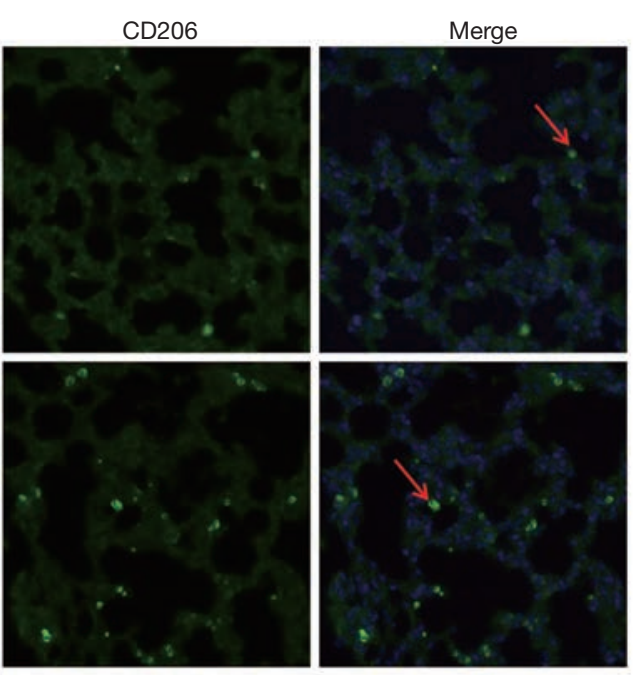
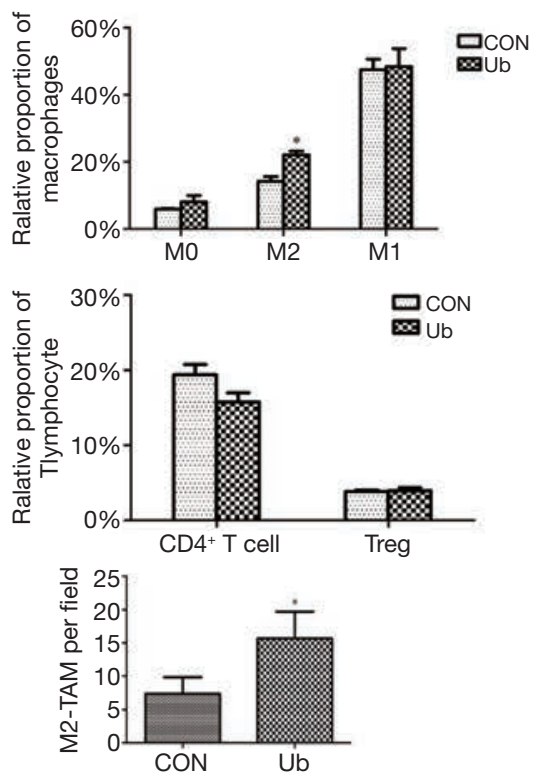

E
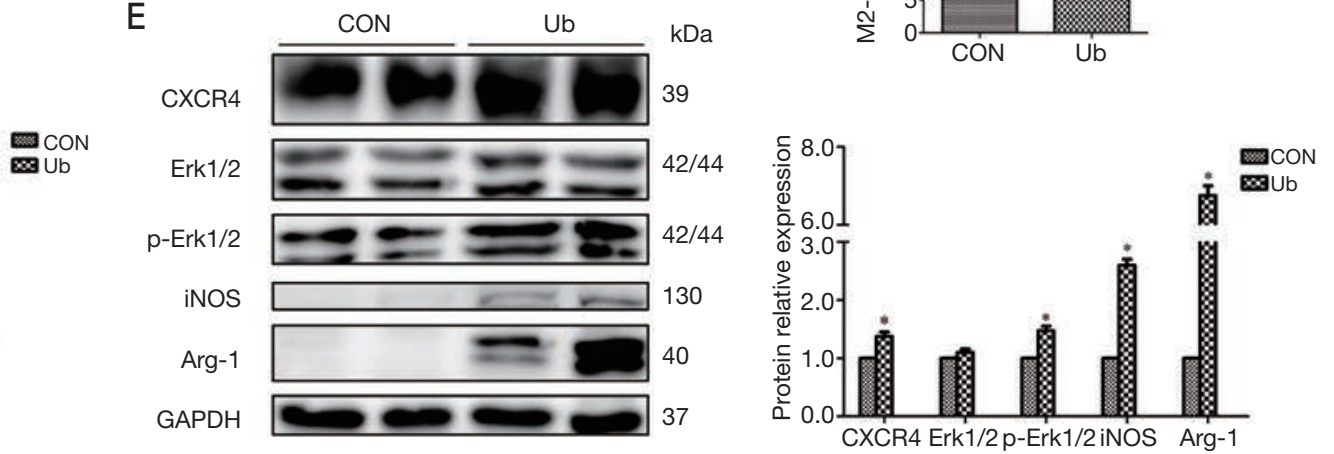

Figure $5 \mathrm{eUb}$ upregulated the ratio of M2 macrophages in tumor-bearing mice. (A,B) The peripheral blood of tumor-bearing mice was collected, and the ratios of macrophages and Tregs were analyzed by flow cytometry. (C) The lung tissues of the tumor-bearing mice were harvested to detect the proportion of M2 macrophages via immunofluorescence assay, the arrows indicated M2 macrophages ( $\times 200)$. (D) The cytokines contained in the serum of tumor-bearing mice were measured by ELISA. (E) Lung tissues were collected to detect the proteins related to macrophage polarization through Western blot. GAPDH was used as a loading control. *, $\mathrm{P}<0.05$. CON, control. 

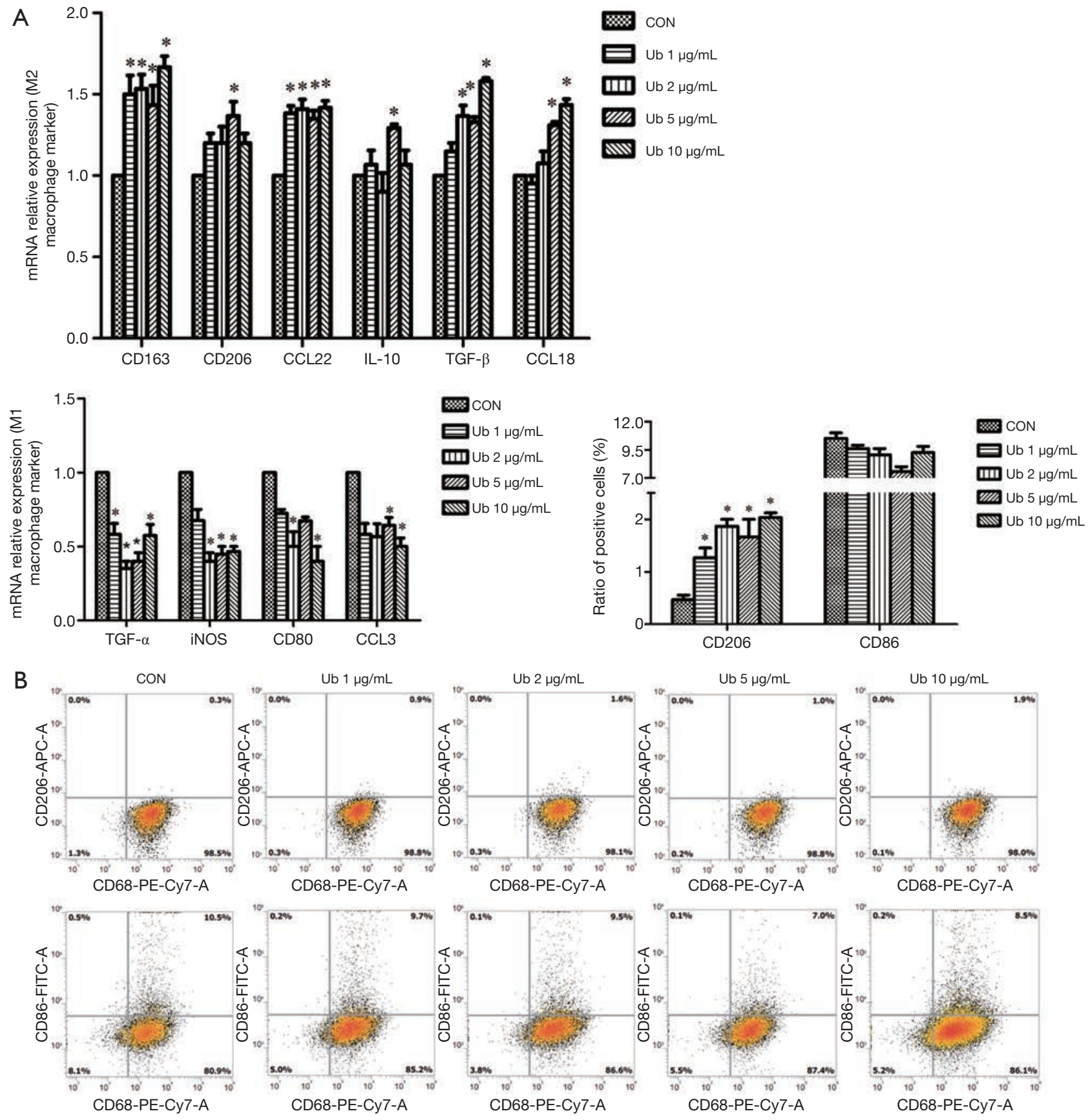

Figure $6 \mathrm{eUb}$ promoted M2 macrophage polarization in vitro. THP-1 monocytes were preincubated with PMA (100 nM) for $24 \mathrm{~h}$ to induce M0 macrophages. After M0 macrophages were treated with eUb $(1,2,5,10 \mu \mathrm{g} / \mathrm{mL})$ for $72 \mathrm{~h}$, the mRNA expressions of macrophage markers were measured by qPCR and normalized by GAPDH expression (A), the expressions of macrophage markers CD206 and CD86 were analyzed by flow cytometry (B). *, $\mathrm{P}<0.05$. CON, control. 
A

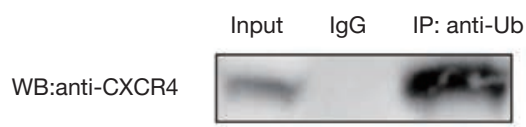

B
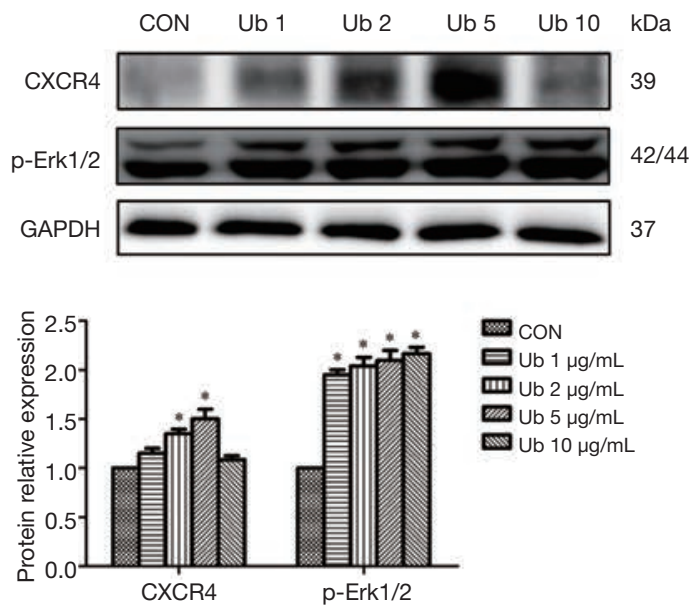

$\mathrm{D}$
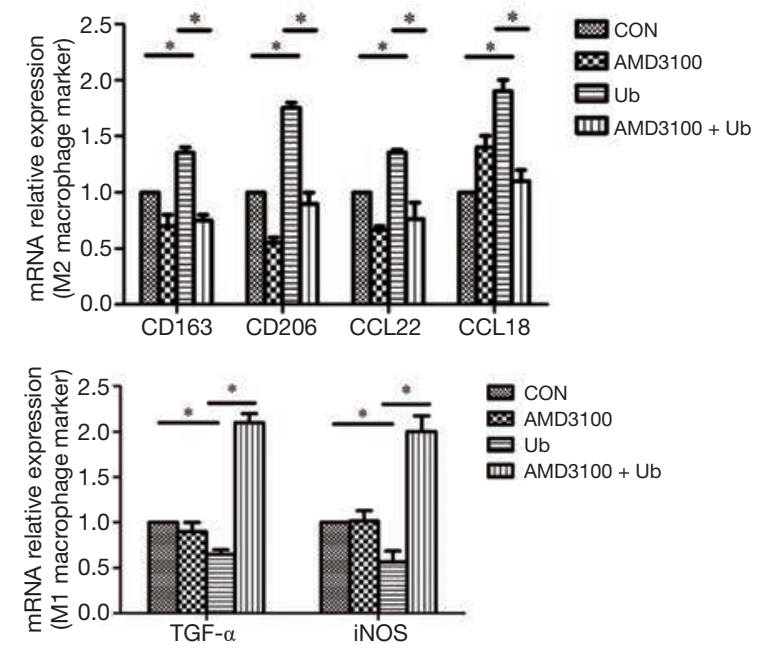

$\mathrm{F}$

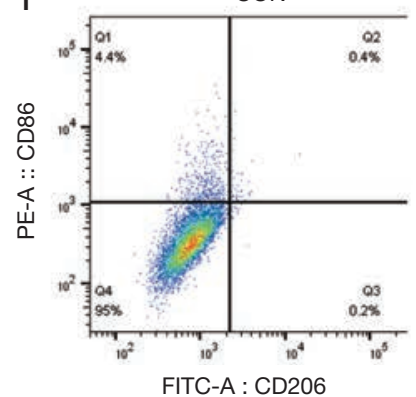

C
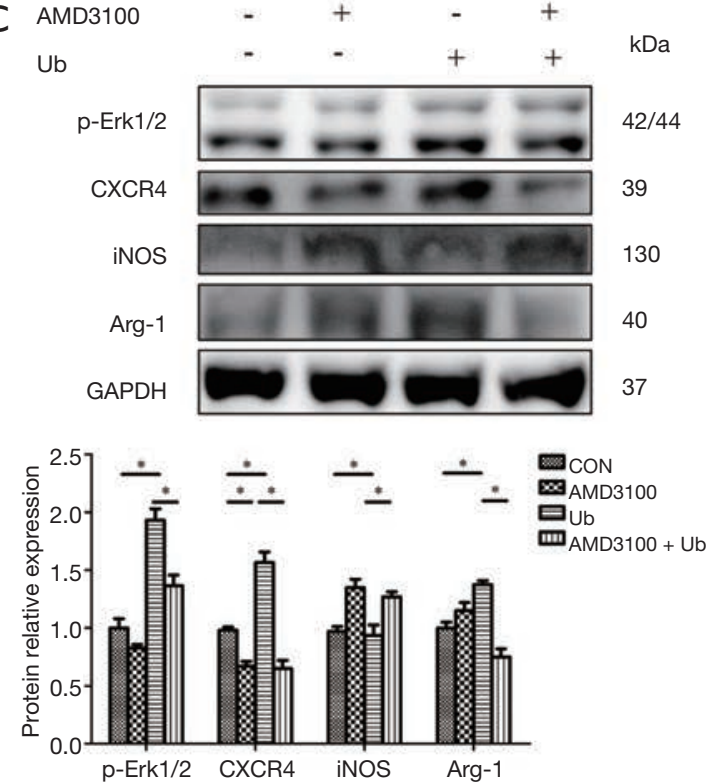

$\mathrm{E}$
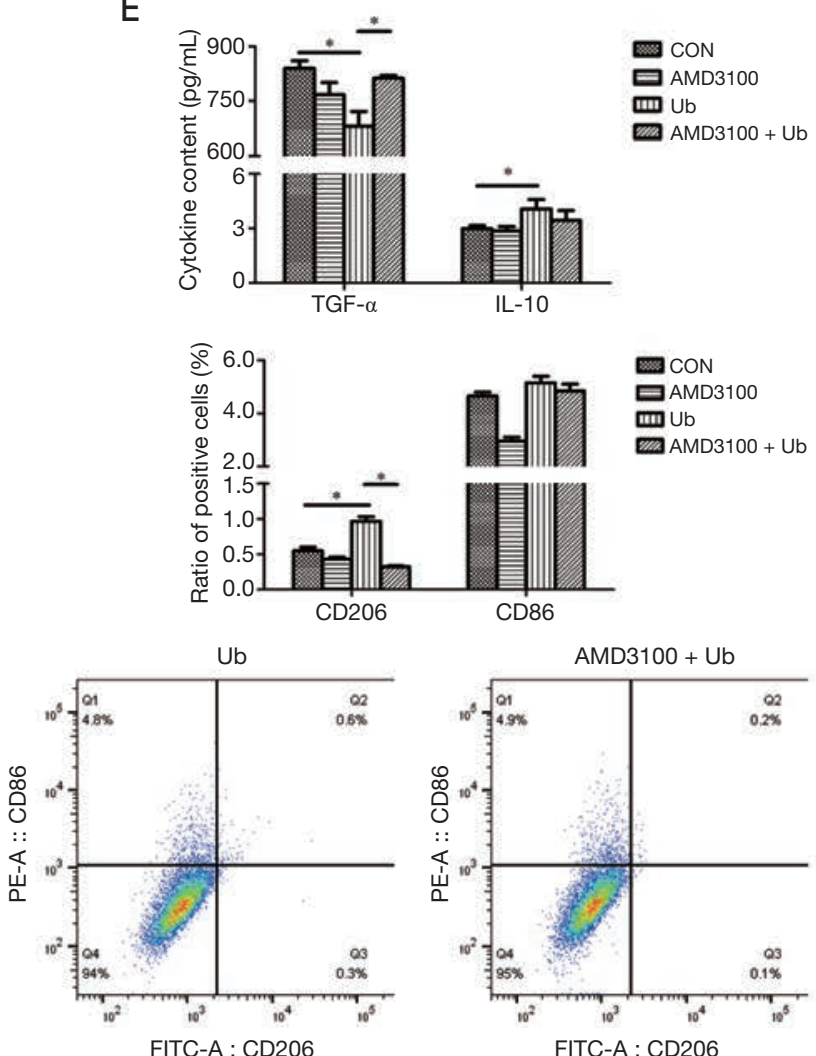

FITC-A : CD206

Figure $7 \mathrm{eUb}$ might promote M2 macrophage polarization via the CXCR4 receptor. (A) The binding of eUb with CXCR4 was confirmed by immunoprecipitation. (B) The protein expressions of CXCR4 and p-Erk1/2 in macrophages after treatment with eUb $(1,2,5,10 \mu \mathrm{g} / \mathrm{mL})$ for $72 \mathrm{~h}$ were detected using Western blot. After pretreatment with AMD3100 $(100 \mu \mathrm{M})$, the M0 macrophages were incubated with eUb $(10 \mu \mathrm{g} / \mathrm{mL})$ for $72 \mathrm{~h}$ and the protein expressions of CXCR4, p-Erk1/2, and macrophage markers (iNOS, Arg-1) were measured by Western blot (C), the mRNA levels of M1 and M2 macrophage markers were detected by qPCR (D), the cytokines in macrophage culture medium were tested by ELISA (E), the expressions of macrophage markers CD206 and CD86 were checked by flow cytometry (F). GAPDH was used as a loading control. *, $\mathrm{P}<0.05$. CON, control. 
p-Erk1/2 were increased in the lung tissues of eUbtreated mice compared with the control mice (Figure $5 E$ ). Therefore, we utilized AMD3100 (CXCR4 inhibitor) and U0126 (Erk1/2 inhibitor) respectively, to explore whether the CXCR4/Erk pathway was involved in eUb-stimulated M2 macrophage polarization.

The M0 macrophages were incubated with eUb $(10 \mu \mathrm{g} / \mathrm{mL})$ for $72 \mathrm{~h}$ after pretreated by AMD3100 $(100 \mu \mathrm{M})$ and U0126 $(10 \mu \mathrm{M})$ for $30 \mathrm{~min}$. The results of Western blot showed that AMD3100 inhibited the eUb-induced increase in the expressions of CXCR4, p-Erk1/2, and Arg-1, in contrast to the alteration of iNOS (Figure 7C). The results of qPCR also showed that the AMD3100 preincubation attenuated the upregulation of M2 macrophage markers (CD163, CD206, CCL22, and CCL18), and stimulated the downregulation of M1 macrophage markers (TNF- $\alpha$ and iNOS) induced by eUb (Figure 7D). Moreover, ELISA analysis showed that the AMD3100 pretreatment upregulated the decrease in TNF- $\alpha$ induced by eUb, but it had no influence on the concentration of IL-10 (Figure 7E). Finally, AMD3100 inhibited the eUb-mediated increase in CD206, while CD86 was not dramatically affected by eUb and AMD3100 exposure (Figure 7F).

Besides, in Western blot analysis, the preincubation of Erk1/2 inhibitor U0126 decreased the upregulation of p-Erk1/2, CXCR4, and Arg-1 and enhanced the downregulation of iNOS induced by eUb (Figure $8 A$ ). The qPCR results showed that U0126 attenuated the upregulation of M2 macrophage markers (CD206, IL-10, TGF- $\beta$, and CCL18) and stimulated the downregulation of M1 macrophage markers (TNF- $\alpha$ and iNOS) induced by eUb (Figure $8 B$ ). Moreover, U0126 reversed the eUbmediated alterations of TNF- $\alpha$ and CD206 but it did not affect the expressions of IL-10 and CD86 in ELISA and flow cytometry analysis, respectively (Figure $8 C, D$ ). Collectively, the above findings indicated that the CXCR4/ Erk pathway was involved in the polarization of macrophage and that by inhibiting the CXCR4/Erk pathway, M2 macrophage polarization stimulated by eUb could be attenuated.

\section{Discussion}

Because of the upregulation of Ub in body fluids seen in many diseases, eUb is identified as a disease biomarker (23). We found that $\mathrm{Ub}$ was elevated in HCC tissues compared with the adjacent nontumor and normal liver tissues, Wang et al. also confirmed that Ub was upregulated in HCC specimens and that the expression of Ub was negatively associated with the clinicopathological degree of HCC, as well as breast cancer, rectal cancer, prostate cancer, and ovarian cancer (24). Meanwhile, CXCR4 was upregulated in HCC tissues, and there was a positive correlation between Ub and CXCR4. CXCR4 is a 7-transmembrane $G$ protein-coupled receptor that is expressed on numerous cells, including cancer cells, lymphocytes, and monocytes (25). CXCR4 was reported to correlate with the proliferation and invasiveness of cancer cells. Yang et al. confirmed the increased expression of CXCR4 in high-grade chondrosarcoma tissues compared with lowgrade specimens and that its expression was closely related with cancer recurrence (26); Jiang et al. also indicated that the high CXCR4 expression in gastric, colorectal, and esophageal cancer could predict a poor prognosis (27). eUb is considered to be an endogenous agonist of CXCR4, and the binding of eUb and CXCR4 initiates $\mathrm{Ca}^{2+}$ influx into the cells and induces chemotactic response (28). Moreover, Ub-CXCR4 interaction was suggested to contribute to lung tumor metastasis induced by acute lung infection (29). Taken together, the pro-tumor effect of eUb could be related to the involvement of CXCR4.

Sudzhashvili et al. demonstrated that eUb injection stimulated the proliferation of hepatectomized alcoholic liver cells (30), while Scofield et al. found that eUb alone had no effect on the proliferation of fibroblasts (31). eUb has also been shown to inhibit the cardiac fibrosis stimulated by $\beta-A R(32)$, and to inhibit the growth of KT-3 and HL-60 cells through the degradation of STAT3 (22). All of these studies suggested that the cell-type-specific roles of eUb are affected by the fundamental pathological state (33). In this study, we found that $\mathrm{eUb}$ had no effect on the migration of hepatoma cells directly in vitro; meanwhile, the metastasis ability of hepatoma cells was enhanced after coculture with macrophages pretreated with eUb, which indicated that $\mathrm{eUb}$ might regulate the function of macrophages to affect hepatoma metastasis.

Tumor development and progression depend on the local tumor microenvironment, which includes lymphocytes, macrophages, monocytes, myofibroblasts, fibroblasts, mesenchymal stem cells, and the extracellular matrix. The macrophages within the tumor microenvironment are termed as tumor-associated macrophages (TAMs), which are crucial regulators of the intricate interaction between cancer and the immune system (34). TAMs are usually divided into two phenotypes: (I) M1 macrophages, which can be induced by LPS alone or in combination with Th1 

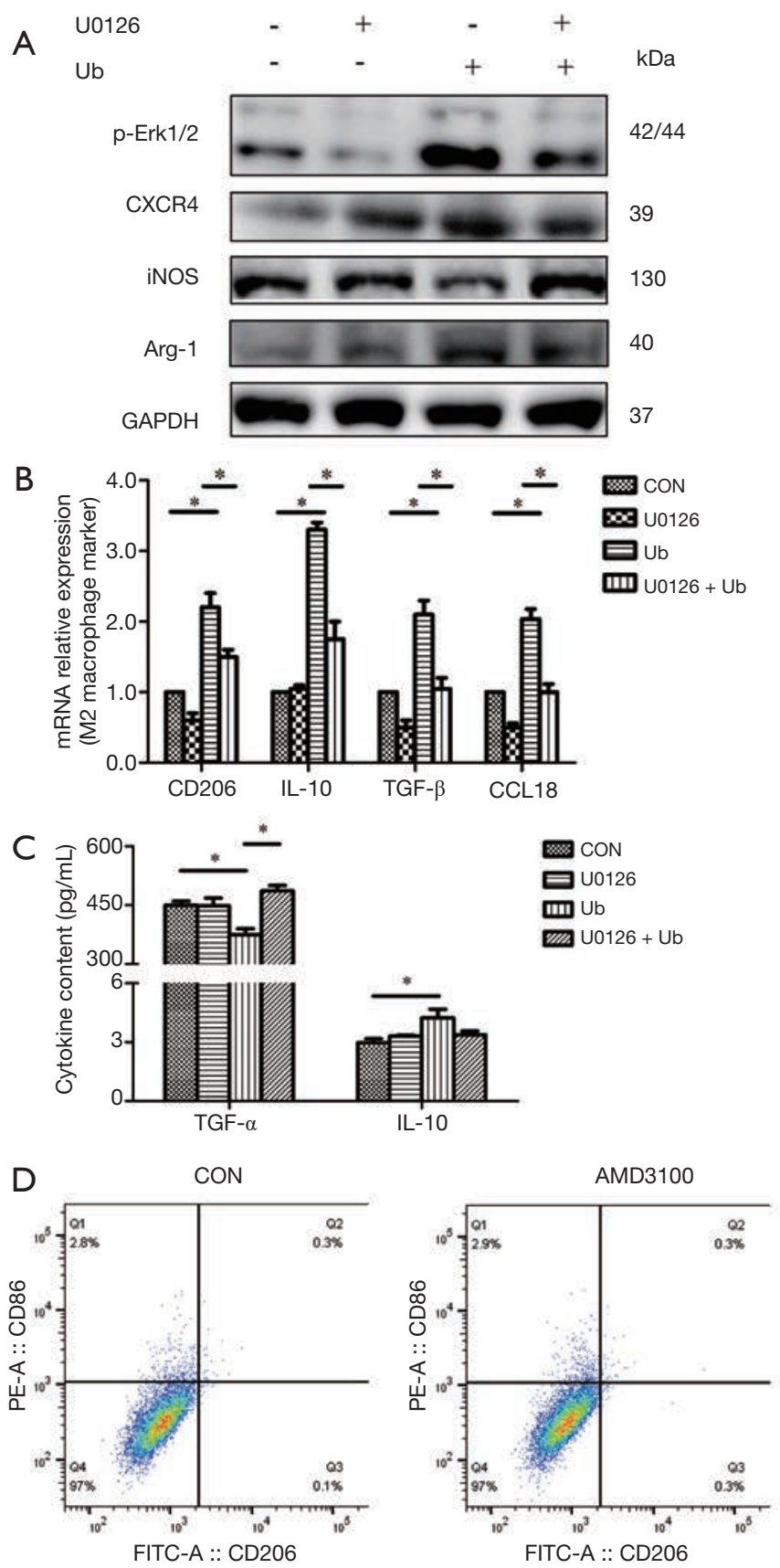
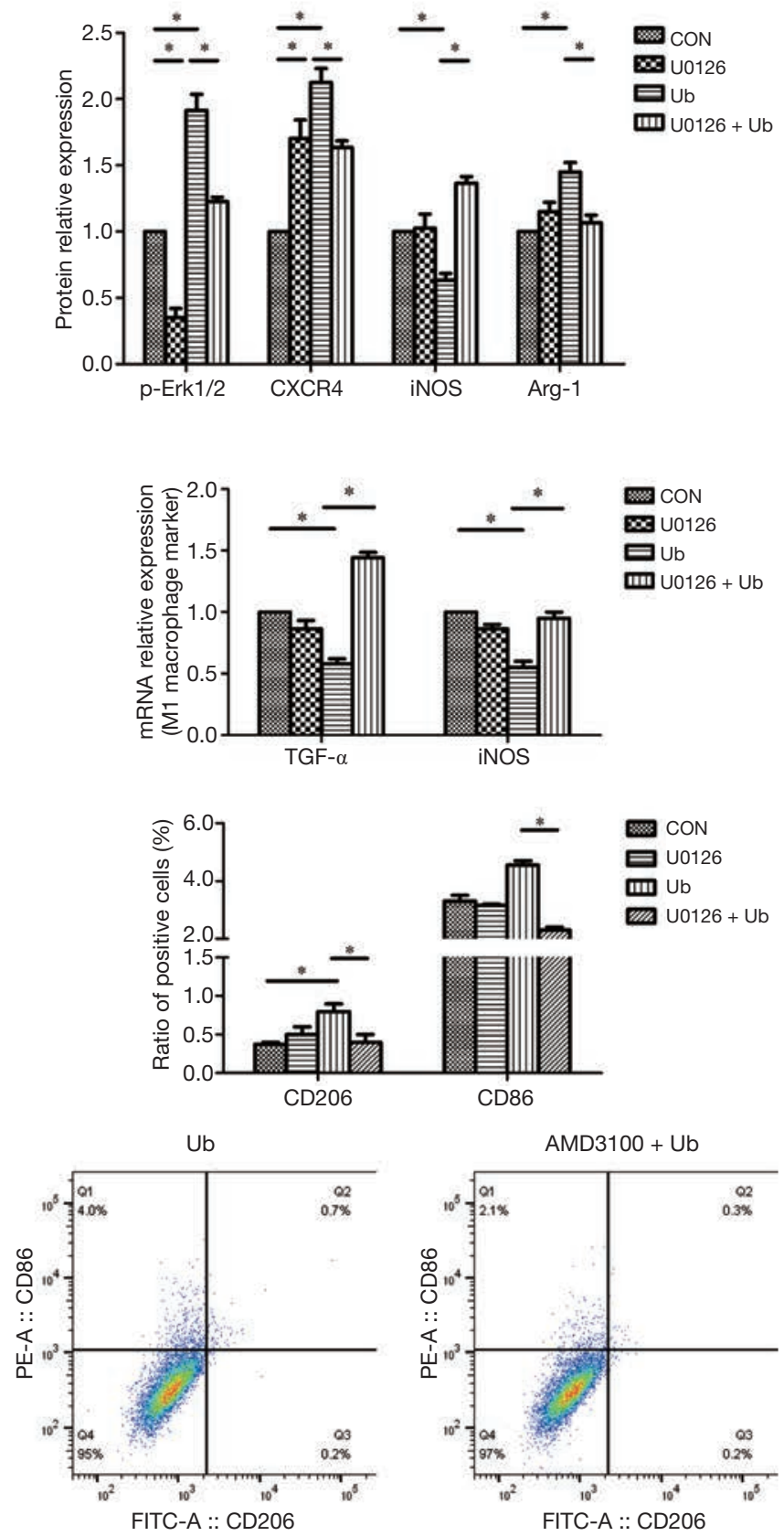

Figure $8 \mathrm{eUb}$ might promote M2 macrophage polarization via Erk1/2 kinase. After U0126 pretreatment (10 $\mu \mathrm{M})$, the M0 macrophages were incubated with $\mathrm{eUb}(10 \mu \mathrm{g} / \mathrm{mL})$ for $72 \mathrm{~h}$ and then the protein expressions of CXCR4, p-Erk1/2 and macrophage markers (iNOS, Arg-1) were measured by Western blot (A), the mRNA levels of M1 and M2 macrophage markers were detected by qPCR (B), the cytokines in macrophage culture medium were tested by ELISA (C), the expressions of macrophage markers CD206 and CD86 were checked by flow cytometry (D). GAPDH was used as a loading control. *, P<0.05. CON, control. 
cytokines such as GM-CSF and IFN- $\gamma$ and secrete proinflammatory cytokines, including TNF- $\alpha$, IL- $1 \beta$, IL-6, and IL-12; and (II) M2 macrophages, which can be induced by Th2 cytokines such as IL-13 and IL- 4 and release antiinflammatory cytokines, including TGF- $\beta$ and IL- 10 . Macrophages can switch from one phenotype to another in a process called "macrophage polarization" (35). Multiple studies have indicated that TAMs in malignant cancers commonly display M2-like phenotypes, which contribute to an immunosuppressive microenvironment and promote tumor development and progression (36). In our study, we not only demonstrated eUb could promote the metastasis of hepatoma cells in vivo but also confirmed the upregulation of M2 macrophages in lung tissues and peripheral blood of tumor-bearing mice after eUb intervention. Furthermore, we found the macrophages displayed M2 phenotype after $\mathrm{eUb}$ treatment in vitro. Therefore, we concluded that $\mathrm{eUb}$ might promote $\mathrm{M} 2$ macrophage polarization to fuel the metastasis of HCC.

The potential underlying mechanism of M2 macrophage polarization mediated by eUb was also explored in vitro. We found CXCR4 and p-Erk1/2 to be elevated after eUb exposure, and the utilization of CXCR4 and Erk1/2 inhibitors blunted the M2 macrophage polarization induced by eUb. Meng et al. reported that the increased p-Erk1/2 expression in the tumor vasculature was related to the higher expression of CXCR4 (37). Extracellular signalregulated protein kinases 1 and 2 (Erk1/2) are serine/ threonine kinases involved in cell growth and differentiation (38). Richardson et al. demonstrated that Erk1/2 exerts a crucial role in driving M-CSF-dependent proliferation of bone marrow-derived macrophages (38). Meanwhile, in breast cancer, the tumor-derived lactate could induce M2 macrophage polarization via the activation of the Erk/STAT3 pathway (34). More importantly, Zhang et al. reported that the MAPKs (mitogen-activated protein kinases) pathway was involved in hypoxia-induced M2 macrophage polarization, and although three MAPKs, JNK, Erk1/2, and p38 were activated under hypoxia, only the Erk1/2 inhibitor repressed the M2 macrophage polarization induced by hypoxia (39). Collectively, we hold the opinion that eUb could activate the CXCR4/ERK pathway to mediate M2 macrophage polarization and promote hepatoma metastasis.

\section{Conclusions}

Our study not only demonstrated that eUb could contribute to the hepatoma metastasis via the involvement of M2 macrophage polarization, but also identified that the activation of CXCR4/ERK signaling pathway could be the underlying mechanism of eUb-mediated M2 macrophage polarization. These findings may provide laboratory evidence of the link between cancer recurrence and TRIM induced by stored RBCs, indicating that personalized transfusion strategies are needed for the treatment of HCC patients. Neutralizing Ub in stored RBC units could lessen the detrimental clinical outcomes induced by stored RBC transfusion.

\section{Acknowledgments}

Funding: This study was supported by the Natural Science Foundation of China (Grant No. 81670173 and 81570166) and Public Health Leading Academic Discipline Project supported by Shanghai Municipal Commission of Health and Family Planning (Grant No. 15GWZK0501).

\section{Footnote}

Reporting Checklist: The authors have completed the ARRIVE reporting checklist. Available at http://dx.doi. org/10.21037/atm-20-1054

Data Sharing Statement: Available at http://dx.doi. org/10.21037/atm-20-1054

Peer Review File: Available at http://dx.doi.org/10.21037/ atm-20-1054

Conflicts of Interest: All authors have completed the ICMJE uniform disclosure form (available at http://dx.doi. org/10.21037/atm-20-1054). The authors have no conflicts of interest to declare.

Ethical Statement: The authors are accountable for all aspects of the work in ensuring that questions related to the accuracy or integrity of any part of the work are appropriately investigated and resolved. All animal protocols were approved by the Laboratory Animal Care and Use Committee of Fudan University (No. 2019 Huashan Hospital JS-259) and conducted under the "Guide for the Care and Use of Laboratory Animals" recommended by the US National Institutes of Health. This study was conducted in accordance with the Declaration of Helsinki (as revised in 2013), and the protocol was approved by the Ethics 
Committee of the Affiliated Hospital of North Sichuan Medical College (No. 2018-EA-028). Informed consent was obtained from each patient.

Open Access Statement: This is an Open Access article distributed in accordance with the Creative Commons Attribution-NonCommercial-NoDerivs 4.0 International License (CC BY-NC-ND 4.0), which permits the noncommercial replication and distribution of the article with the strict proviso that no changes or edits are made and the original work is properly cited (including links to both the formal publication through the relevant DOI and the license). See: https://creativecommons.org/licenses/by-nc-nd/4.0/.

\section{References}

1. Lelubre C, Vincent JL. Relationship between red cell storage duration and outcomes in adults receiving red cell transfusions: a systematic review. Crit Care 2013;17:R66.

2. Koch CG, Li L, Sessler DI, et al. Duration of red-cell storage and complications after cardiac surgery. $\mathrm{N} \mathrm{Engl} \mathrm{J}$ Med 2008;358:1229-39.

3. van Straten AH, Soliman Hamad MA, van Zundert AA, et al. Effect of duration of red blood cell storage on early and late mortality after coronary artery bypass grafting. J Thorac Cardiovasc Surg 2011;141:231-7.

4. Goubran H, Sheridan D, Radosevic J, et al. Transfusionrelated immunomodulation and cancer. Transfus Apher Sci 2017;56:336-40.

5. Cata JP, Wang H, Gottumukkala V, et al. Inflammatory response, immunosuppression, and cancer recurrence after perioperative blood transfusions. Br J Anaesth 2013;110:690-701.

6. Abu-Ghanem Y, Zilberman DE, Dotan Z, et al. Perioperative blood transfusion adversely affects prognosis after nephrectomy for renal cell carcinoma. Urol Oncol 2018;36:12.e15-12.e20.

7. Kneuertz PJ, Patel SH, Chu CK, et al. Effects of perioperative red blood cell transfusion on disease recurrence and survival after pancreaticoduodenectomy for ductal adenocarcinoma. Ann Surg Oncol 2011;18:1327-34.

8. Wang CC, Iyer SG, Low JK, et al. Perioperative factors affecting long-term outcomes of 473 consecutive patients undergoing hepatectomy for hepatocellular carcinoma. Ann Surg Oncol 2009;16:1832-42.

9. Squires MH 3rd, Kooby DA, Poultsides GA, et al. Effect of Perioperative Transfusion on Recurrence and Survival after Gastric Cancer Resection: a 7-Institution Analysis of
765 Patients from the US Gastric Cancer Collaborative. J Am Coll Surg 2015;221:767-77.

10. Haas AL. Ubiquitin-mediated processes in erythroid cell maturation. Adv Exp Med Biol 1991;307:191-205.

11. Takada K, Nasu H, Hibi N, et al. Serum concentrations of free ubiquitin and multiubiquitin chains. Clin Chem 1997;43:1188-95.

12. Haas AL, Wilkinson KD. The large scale purification of ubiquitin from human erythrocytes. Prep Biochem 1985;15:49-60.

13. Patel MB, Proctor KG, Majetschak M. Extracellular ubiquitin increases in packed red blood cell units during storage. J Surg Res 2006;135:226-32.

14. Zhu X, Yu B, You P, et al. Ubiquitin released in the plasma of whole blood during storage promotes mRNA expression of Th2 cytokines and Th2-inducing transcription factors. Transfus Apher Sci 2012;47:305-11.

15. Goldberg AL. Protein degradation and protection against misfolded or damaged proteins. Nature 2003;426:895-9.

16. Cao Y, Li C, Zhang Q, et al. Extracellular ubiquitin enhances the suppressive effects of regulatory $\mathrm{T}$ cells on effector T cell responses. Clin Lab 2014;60:1983-91.

17. Majetschak M, Krehmeier U, Bardenheuer M, et al. Extracellular ubiquitin inhibits the TNF-alpha response to endotoxin in peripheral blood mononuclear cells and regulates endotoxin hyporesponsiveness in critical illness. Blood 2003;101:1882-90.

18. Majetschak M, Cohn SM, Nelson JA, et al. Effects of exogenous ubiquitin in lethal endotoxemia. Surgery 2004;135:536-43.

19. Pancré V, Pierce RJ, Fournier F, et al. Effect of ubiquitin on platelet functions: possible identity with platelet activity suppressive lymphokine (PASL). Eur J Immunol 1991;21:2735-41.

20. Scofield SL, Amin P, Singh M, et al. Extracellular Ubiquitin: Role in Myocyte Apoptosis and Myocardial Remodeling. Compr Physiol 2015;6:527-60.

21. Daino H, Matsumura I, Takada K, et al. Induction of apoptosis by extracellular ubiquitin in human hematopoietic cells: possible involvement of STAT3 degradation by proteasome pathway in interleukin 6-dependent hematopoietic cells. Blood 2000;95:2577-85.

22. Zhang J, Chen S, Yan Y, et al. Extracellular Ubiquitin is the Causal Link between Stored Blood Transfusion Therapy and Tumor Progression in a Melanoma Mouse Model. J Cancer 2019;10:2822-35.

23. Sujashvili R. Advantages of Extracellular Ubiquitin in Modulation of Immune Responses. Mediators Inflamm 
2016;2016:4190390.

24. Wang J, Liu Y, Jiang X, et al. Expression of ubiquitin and P27 protein in hepatocellular carcinoma and its clinicopathlogic significance (in Chinese). Journal of Shanxi Medical University 2010;41:116-9.

25. Saini V, Marchese A, Majetschak M. CXC chemokine receptor 4 is a cell surface receptor for extracellular ubiquitin. J Biol Chem 2010;285:15566-76.

26. Yang P, Wang G, Huo H, et al. SDF-1/CXCR4 signaling up-regulates survivin to regulate human sacral chondrosarcoma cell cycle and epithelial-mesenchymal transition via ERK and PI3K/AKT pathway. Med Oncol 2015;32:377.

27. Jiang Q, Sun Y, Liu X. CXCR4 as a prognostic biomarker in gastrointestinal cancer: a meta-analysis. Biomarkers 2019;24:510-6.

28. Saini V, Staren DM, Ziarek JJ, et al. The CXC chemokine receptor 4 ligands ubiquitin and stromal cell-derived factor- $1 \alpha$ function through distinct receptor interactions. J Biol Chem 2011;286:33466-77.

29. Yan L, Cai Q, Xu Y. The ubiquitin-CXCR4 axis plays an important role in acute lung infection-enhanced lung tumor metastasis. Clin Cancer Res 2013;19:4706-16.

30. Sudzhashvili RSh, Bakuradze ED, Modebadze IR, Dekanoidze DM. Ubiquitin in combination with alcohol stimulates proliferative activity of hepatocytes. Georgian Med News 2013;(223):86-90.

31. Scofield SLC, Daniels CR, Dalal S, et al. Extracellular ubiquitin modulates cardiac fibroblast phenotype and function via its interaction with CXCR4. Life Sci 2018;211:8-16.

Cite this article as: Cai J, Zhang Q, Qian X, Li J, Qi Q, Sun R, Han J, Zhu X, Xie M, Guo X, Xia R. Extracellular ubiquitin promotes hepatoma metastasis by mediating M2 macrophage polarization via the activation of the CXCR4/ERK signaling pathway. Ann Transl Med 2020;8(15):929. doi: 10.21037/atm20-1054
32. Daniels CR, Foster CR, Yakoob S, et al. Exogenous ubiquitin modulates chronic -adrenergic receptorstimulated myocardial remodeling: role in Akt activity and matrix metalloproteinase expression. Am J Physiol Heart Circ Physiol 2012;303:H1459-68.

33. Steagall RJ, Daniels CR, Dalal S, et al. Extracellular ubiquitin increases expression of angiogenic molecules and stimulates angiogenesis in cardiac microvascular endothelial cells. Microcirculation 2014;21:324-32.

34. Mu X, Shi W, Xu Y, et al. Tumor-derived lactate induces M2 macrophage polarization via the activation of the ERK/STAT3 signaling pathway in breast cancer. Cell Cycle 2018;17:428-38.

35. Shapouri-Moghaddam A, Mohammadian S, Vazini H, et al. Macrophage plasticity, polarization, and function in health and disease. J Cell Physiol 2018;233:6425-40.

36. Liu H, Dong H, Jiang L, et al. Bleomycin inhibits proliferation and induces apoptosis in TPC-1 cells through reversing M2-macrophages polarization. Oncol Lett 2018;16:3858-66.

37. Meng YM, Liang J, Wu C, et al. Monocytes/Macrophages promote vascular CXCR4 expression via the ERK pathway in hepatocellular carcinoma. Oncoimmunology 2017;7:e1408745.

38. Richardson ET, Shukla S, Nagy N, et al. ERK Signaling Is Essential for Macrophage Development. PLoS One 2015;10:e0140064.

39. Zhang J, Cao J, Ma S, et al. Tumor hypoxia enhances Non-Small Cell Lung Cancer metastasis by selectively promoting macrophage M2 polarization through the activation of ERK signaling. Oncotarget 2014;5:9664-77. 